M. Georges Bouvet, directeur du Jardin botanique d'Angers, auquel on doit de nombreux Mémoires sur la flore de l'Ouest.

En conséquence, la liste composée des noms de MM. Hr, Gadecenu el Bouvet est proposée, au nom du Conseil d'administration, aux suffrages de la Société, et elle est adoptée par l'assemblée à une grande majorité. Elle sera immédiatement notifiée à M. le Maire d'Angers.

M. Gagnepain, vice-secrétaire, donne lecture de la communication suivante :

SUR LA VÉGÉTATION DE QUELQUES SOURCES D'EAU DOUCE SOUS-MARINES DE LA SEINE-INFÉRIEURE;

par M. Mauriee GomovT.

Peu de pays sont aussi connus, au moins des habitants du nord de ?a France, que la région côtière du département de la SeineInférieure, dont les nombreuses stations de bains de mer attirent chaque été une foule de visiteurs. On sait qu'elle se compose d'un vaste plateau connu sous le nom de pays de Caux, se terminant brusquement du côté de la mer par une muraille verticale ou falaise. Le sol de ce plateau est formé, comme son nom l'indique (1), de puissantes assises calcaires que recouvrent des dépôts de limon, de sable et d'argile. Il est, en général, perméable aux eaux pluviales sur une grande épaisseur, aussi les sources y sontelles rares, surtout le long des côtes, et, pour y suppléer, les habitants sont-ils forcés de recourir à des citernes ou à des puits profonds.

En parcourant le rivage on remarque que ces sources, absentes sur le plateau, apparaissent en revanche nombreuses au niveau supérieur des marées ou au-dessous. Les unes, émergeant au pied mème des falaises, sont connues et utilisées depuis longtemps, tandis que les autres, moins accessibles et restant moins longtemps à découvert, sont habituellement peu remarquées. D'après les traditions locales, certaines d'entre elles ne seraient que le

(1) Caux, Causse, du latin culx, chaux. 
GOMONT. - VÉGÉTATION DES SOURCES D'EAU DOUCE SOUS-MARINES. 37

débouché dans la mer d'anciennes rivières qui auraient disparu de la surface du sol en se frayant un passage par les nombreuses fissures des couches calcaires (1). Il se passe donc ici, sur une moindre échelle, le même phénomène que sur les Causses du midi de la France où l'eau du ciel, absorbée par les fentes et les gouffres naturels, reparait dans les vallées sous forme de sources puissantes. Seulement, dans le cas dont il s'agit, la vallée, c'est la Manche, et des plaines d'une fertilité renommée remplacent le Causse rocailleux et stérile.

Ayant eu l'occasion de parcourir fréquemment la partie de la côte comprise entre Saint-Valery-en-Caux et Étretat, j’ai reconnu que ces courants d'eau douce, dont quelques-uns sont abondants, apportaient des modifications nolables dans la végétation marine. Le fait n'a rien qui puisse surprendre. Tous ceux qui ont herborisé à la mer connaissent les changements qu'amène dans la Flore marine le voisinage des estuaires, et M. Oltmanns en a indiqué les causes dans un Mémoire sur lequel j'aurai l'occasion de revenir (2). Toutefois l'auteur n'a donné sur ce sujet que des indications générales, ou peu s'en faut, et n'a pas étudié l'énergie de cette influence sur les diverses espèces d'une région déterminée. De son còté, Debray, dans sa Florule marine du nord de la France, cite à peine ce genre de localités (3). La question est donc restée à peu près intacte et je pense qu'il ne sera pas sans intérêt de jeter un coup d'œil sur la topographie et la végétation de certaines de ces sources que j'ai été à même de visiter à plusieurs reprises et à des époques différentes de l'année. Rappelons d'abord la configuration de la côte oủ elles se rencontrent et le peuplement végétal des plages environnantes.

Vue du large, la partie de la côte normande qui s'étend du Tréport au Havre apparaìt comme un rempart vertical, à tracé sinueux, dont la hauteur varie de 70 à 100 mètres. Il est ébréché

(1) Ces cours d'eau auraient disparu, dans tous les cas, avant le milieu du xvirl $^{e}$ siècle, attendu que la carte de Cassini, qui date de cette époque, ne figure aucun autre cours d'eau que ceux qui existent de nos jours.

(2) Oltmanns, Ueber die Bedeutung der Concentrations-änderungen des Merwassers für das Leben der Algen, in Sitzungsberichte der $\boldsymbol{K}$. preussischen Akademie der Wissenschaften zu Berlin, 1891, pp. 193-203.

(3) Conf. Debray, Florule des Algues marines du Nord de la France, in Bulletin scientifique de la France et de la Belgique, t. XXXII, 1899, pp. 43, 103 et 143. 
de place en place par des vallées, la plupart desséchées, du moins à Y'époque actuelle, quelques-unes arrosées par des rivières qui se rendent directement à la mer. Cette coupe naturelle met à nu de puissantes couches crétacées d'autant plus anciennes qu'on se rapproche davantage de l'embonchure de la Seine et désignées par les géologues sous les noms de Craie blanche, Craie marneuse, Craie glaueonieuse. A Saint-Jouin, non loin du Havre, elle alteint le Gault et entame même, à la Hève, la partie supérieure des terrains jurassiques. La couleur de ces assises calcaires varie du blanc pur au gris, sauf dans les endroits où elles sont salies par des éboulements argileux provenant de dépòts supérieurs à la craie. Elles sont rayées horizontalement de lignes foncées, souvent très rapproehées, qui représentent de minces lits de silex alternant avee les couches crayeuses.

L'effet des vagues sur ces falaises est connu de tous. Elles en minent la base, soit chaque jour, soit seulement aux grandes marées, suivant les points considérés. De temps à autre un pan de la muraille s'écroule et ses fragments sont allaqués par les flots. La craie, plus tendre, se délite, laissant à nu le silex, dont les fragments sont façonnès en galets par le va-et-vient continuel de la mer.

La confignration du rivage qui a été produit par ce travail des vagues continué pendant une longue suite de siècles, est partout la même. A la base des falaises règne un talus de galets siliceux assez incliné et sensiblement divisé en terrasses. Continuellement roulés et remaniés par la mer, les matériaux dont il est formé ne peuvent donner prise à aucune végétation; aus:i cette région est-elle complètement stérile, sauf sur quelques blocs détachés où se rencontrent les espèces qui se plaisent à la limite supérieure des marées.

A partir du bas de ce talus, et aussi loin que le niveau de la basse mer permet à la vue de s'étendre, règnent des bancs de rochers presque horizontaux. La eraie s'y montre le plus souvent à la surface, mais çà et là, elle est recouverte par des lits de silex simplement fissurés ou par des amas de cailloux de même nature, irréguliers et caverneux, entre lesquels le pied a peine à se poser. Là encore la végétation est à peu près nulle, mais, partoul ailleurs, s'étend un tapis de Fucus mêlés à d'autres espèces moins apparentes, qui donne au rivage, à basse mer, une 
couleur noire earactérístique. De loin, ces banes rocheux sembłent former une surface à peu près plane, mais, ainsi que l'a fait remarquer Debray (1), ils sont, en réalité, sillonmés de profondes crevasses à parois verticales, qui s'étendent souvent sur plusieurs centaines de mètres et toujours perpendiculairement à la ligne des côtes. A marée montante, les vagues s'y engouffrent avec violence, surtout quand elles sont poussées par les vents du large, de sorte que la végétation de ces canaux est assez pauvre.

Enfin, sur certains points du rivage, ces banes horizontaux sont parsemés de gros rochers, dont l'origine et la nature minéralogique sont tout autres. Ge sont des bloes de grès éboulés dio haut des falaises, oú on les trouve en place parmi des couches de sable et de limon appartenant à une formation plus récente.

Il serait inutile pour le but que je me propose de donmer ume liste complète des Algues pouvant se rencontrer dans la région que j’ai explorée; on la trouvera d'ailleurs dans l'ouvrage de Debray cité plus haut. En effet, étant donnée la faible surfaeé baignée par les sources, le manque d'une espèce qui n'est pas répandue partout pourrait toujours être attribué ả une autre cause qu'à l'influence de l'eau douce. Je me bornerai donc à énumérer les plantes qui forment le fond de la végétation, en suivant autant que possible leur ordre de fréquence dans la région.

Les trois familles qui occupent la plus grande surface, au point. de vue du nombre des individus, sont, sans contredit, les Fucacées, les Ulvacées et les Corallines. Les Fucacées, comme je l'ai dit plus haut, revêtent d'un tapis uniforme les bancs de rochers qui s'étendent, à peine interrompus par quelques plages de sable ou de vase, entre le talus de galets et la limite de la basse mer. Les Fucus platycarpus, serratus et vesiculosus les représentent à peu près exclusivement. Lè Fucus ceranoides, aussi bien que le Pelvetia canaliculata, paraît rare dans la région; l'Halidrys siliquosa n'existe qu'en individus isolés dans les llaques profondes. Quant anx genres Ascophyllum, Himanthalia et Cystosira, je n'en ấ rencontré aucun échantillon, du moins en place.

Les Ulvacées, représentées par l'Enteromorpha intestinalis e quelques autres, ainsi que par l'Elva Lactuca, sont très abondantes dans la zone supérieure des marées et descendent dans lat

(†) F. Debray; loco cit., p. 7 . 
zone moyenne, mêlées aux Fucus de plus en plus nombreux vers la haute mer. L'Enteromorpha intestinalis, en particulier, forme une lisière verte très apparente au bas du talus de galets, lá oủ le rivage devient horizontal; sur les points oủ il rencontre l'eau douce ou saumâtre, il se développe encore plus vigoureusement.

Enfin le Corallina officinalis tapisse presque partout les parois des flaques et des trous creusés dans le rocher, associé à divers Lithothamnion.

En fait de Laminaires, je n'ai vu que les $L$. saccharina et flexicaulis et encore étaient-ils jetés à la còte à la suite des tempètes. C'est seulement à Fécamp que j'ai rencontré en place le L. flexicaulis. Il couvre là de grandes surfaces, oủ il se substitue aux Fucus. D'après Debray, il s'étendrait jusqu'au Havre.

Voici d'ailleurs la liste, par ordre de fréquence, des plantes qu'on peut regarder comme formant le fond de la végétation, attendu qu'on les rencontre, soit à peu près partout, soit en abondance aux différents niveaux qui leur conviennent.

Fucus platycarpus.

- serratus.

- vesiculosus.

Enteromorpha intestinalis.

Uiva lactuca.

Corallina officinalis.

Ceranium rubrum.

Chondrus crispus

Gigartina mamillosa.

Rhodochorton floridulum.

Laurencia pinnatifida.

Plocamium coccineum.

Cladophora rupestris.

Porphyra laciniata.

Dictyota dichotoma.

Nitophyllum laceratum.

Griffithsia setacea.
Cystoclonium purpurascens.

Gelidium crınale.

- corneum.

Polysiphonia nigrescens.

Spermothamnion Turneri.

Polyides rotundus.

Cladophora utriculosa Kütz. (C. leelevirens Harvey).

Lomentaria articulata.

Delesseria Hypoglossum.

- alata.

Ceramium Deslongchampsii.

- echionotum.

Callithamnion tetricum.

Rhodymenia palmatit.

- Palmetta.

Elachistea fucicola.

Comme on le voit, si on mel de côté les genres Fucus, Ulva et Enteromorpha, qui renferment seulement cinq espèces vulgaires, mais occupent une place exceptionnelle au point de vue du nombre des individus, les Floridées dominent. Elles comptent en effet vingt-trois espèces, alors que les Chlorophycées et les Phæophycées réunies en comptent quatre seulement et les Myxophycées aucune. Un fait qui frappe également, quand on explore cette région, c'est le petit nombre des Eclocarpus, qui 
GOMONT. - VÉGÉTATION DES SOURGES D'EAU DOUGE SOUS-MARINES. 41 abondent au contraire, aussi bien que les Dictyotées, de l'autre côté de la baie de la Seine, sur les rochers du Calvados.

Examinons maintenant ce que devient cette végétation sous l'influence de l'eau douce. Dans ce but, je passerai successivement en revue les différentes sources que j'ai étudiées, en suivant la côte du nord-est au sud-ouest, de Saint-Valery-en-Caux jusqu'à Étretat.

\section{Source $\mathrm{n}^{\circ} 1$.}

Si, partant de Saint-Valery, on contourne la pointe élevée qui porte le sémaphore, on entre dans une petite baie, ou plutòt une sinuosité de la côte. vers le fond de laquelle se trouve une source, entre la bande littorale de galets et le niveau de la basse mer. L'aspect tout particulier du terrain environnant la fait assez facilement découvrir. Elle sort de terre vers le milieu d'une petite plate-forme un peu surélevée, composée de sable vaseux compact, mêlé de débris de coquilles, qui semble être une sorte de tuf produit en partie par les dépôts de la source elle-même. Je n'ai pu visiter qu'une seule fois, en août 1903, cette localité très éloignée de mon centre d'excursions.

Au moment de ma visite, la plate-forme arrosée par l'eau douce portait pour toute régétation des touffes luxuriantes de Cladophora rupestris, plante que nous retrouverons partout dans ce genre de localités. La source elle-même était, à ce moment, peu abondante et les filets d'eau qui en découlaient se mèlaient presque immédiatement aux flaques marines environnantes, sans former dans la direction de la mer un courant bien déterminé. Il est remarquable qu'une si faible quantité d'eau douce suffise pour écarter de sa sphère d'action toute autre espèce que celle que nous avons citée.

\section{Source $\mathbf{n}^{\circ} 2$.}

En continuant à suivre le rivage dans la même direction, on parvient à un lieu désigné par les habitants du pays sous le nom de Trou au vin et caractérisé, dans l'état actuel de la falaise, par un éboulement considérable d'argile (1). Là existe une source

(1) Gette dénomination vient, paraît-il, d'une excavation de couleur violacée qui existait autrefois sur ce point de la falaise. Elle a disparu à la suite 
abondante qui formait une véritable rivière quand je l'ai visitée pour la première fois, en 1899. Pendant les années sèches, son débit avait un peu diminué, mais je ne l'ai jamais vue tarir. Elle sort, par deux orifices, à 200 mètres environ du niveau supérieur des marées, mais il en existe évidemment d'autres dans le lit même du ruisseau, car celui-ci grossit notablement en descendant vers la mer, sans que l'eau m'ait paru devenir saumâtre.

L'eau douce coule d'abord sur des bancs horizontaux de pierre siliceuse, recouverts d'un enduit blanchàtre, puis sur un lit de sable parsemé de blocs. La largeur du courant est, en moyenne, de 20 mètres. Cette eau est très limpide, un peu plus froide que l'eau de la mer et surtout que celle des flaques et des petits courants d'eau salée environnants. La profondeur du courant est naturellement très variabie, mais il est impossible à traverser sans que l'eau atteigne la hauteur du genou.

Sur une longueur de $\mathbf{1 0}$ mètres environ à partir du point où sort l'eau douce, s'étend une véritable prairie verdoyante d' $\boldsymbol{E} \boldsymbol{n}$ teromorpha intestinalis qui garnit toutes les fissures du banc siliceux et tous les points où l'Algue peut se fixer (1). Plus bas, sur le lit de sable, la végétation se réduit à de grosses touffes de Gigartina mamillosa, de Chondrus crispus et de Ceramium rubrum.

Au mème endroit se rencentre également le Callithamnion Hookeri, parasite sur le Ceramium comme sur le Gigarlina, et je dois faire remarquer que, sur cette côte, je l'ai rençontré seu-

de l'éboulement dont nous voyons actuellement la trace. Peut-être cette cotoration insolite était-elle due à la présence d'une Chroococacée. Souvent, en effet, elles couvrent ici la craie sur de grandes surfaces.

(1) Cette abondante végétation d'Enteromorpha se retrouve à l'embouchure de la Durdent, à quelques kilomètres vers le Sud-Ouest. Avant d'arriver à la mer, cette rivière rencontre une épaisse digue naturelle de galets et la traverse sous un tunnel au débouché duquel est établie une valve, sorte de soupape de grandes dimensions qui s'ouvre au moment du reflux pour donner passage au courant et se ferme sous la pression de la marée montante, interdisant ainsi à la mer l'accès de la valfée. $\Lambda$ marée basse la Durdeut descend en formant urr courant impétueux dont le lit est complètement tapissé d' $\mathbf{E} n$ teromorpha intestinalis comme eelui de la source dont nous parlens. D'autre part on retrouve la même plante en non moius grande abondance en amont de la valve, où l'eau de mer ne pénètre pas. Nous venons de voir d'ailleurs qu'elle croît tout le long du rivage, à la limite supérieure des marées. On doit en conclure que, si cette Algue préfêre les eaux saumâtres, elle jouit cependant, au point de vue de la safure, d'une très grande tolérance. 
lement dans les courants d'eau douce. Aucun auteur, que je sache, n’a fait mention de celte préférence.

Voici la liste de toutes les espèces dont la présence a été constatée dans la source $n^{\circ} 2$ et dans le ruisseau qui en sort.

Enteromorpha intestinalis.

Chondrus crispus.

Gigartina mamillosa.

Ceranium rubrum.

Ulva Lactuca.
Cladophora rupestris.

Porphyra laciniata.

Callithamnion Hookeri.

Ceramium Deslongehampsii.

Polysiphonia nigreseens.

Les deux dernières plantes n'ont été trouvées qu'en échantillons uniques; je pense done qu'on peut regarder leur présence comme accidentelle.

Il importe de remarquer que, sur cette liste, ne figurent ni Fucus, ni Gorallines, bien que ces plantes existent en abondance dans le voisinage. Si on rencontre quelques touffes de Fucus, elles sont constamment fixées sur des pierres émergées; hors de l'atteinte de l'eau douce. Il m'a paru que c'était un fait général, ne souffrant, dans ces conditions de milieu, que de très rares exceptions.

\section{Source $\mathbf{n}^{\circ} \mathbf{3}$.}

A quelque distance de la source précédente, en s'éloignant de Saint-Valery, il en existe une antre qui sort au pied même du cordon littoral de galets, à peu de distance par conséquent audessous du niveau supérieur des marées. Bien que d'un faible volume relativement à la précédente, elle donne également lieu à un développement considérable d'Ulva Lactuca et d'Enteromarpha intestinalis. Au point où sort l'eau douce, ces deux espèces composent même toute la végétation. Un peu plus bas, l'eau donce se mélange à celle des flaques laissées par la mer et se trouve envahie par la végétation ambiante. Je mentionne cette source, encore qu'elle soit de faible importance, parce qu'elle appartient à un type particulier, celui des épanehements d'eau douce qui se produisent au nivean du cordon littoral. 


\section{Source $\mathbf{n}^{\circ} 4$.}

Elle est située à peu de distance vers le nord-est du vallon de Sunsette (1), où aucun ruisseau n'existe actuellement, et sort de terre à cent dix mètres environ du niveau supérieur des marées, au milieu de l'enceinte de perches d'une ancienne pècherie. Sur ce point, comme à la source $\mathbf{n}^{\circ} \mathbf{1}$, le sol se trouve rehaussé par une succession de petites terrasses formées de dépôts calcaires, meubles à la surface, et mélangés de sable siliceux. Plus loin, ce limon disparait et le ruisseau coule sur un lit de calcaire dur et poli, parsemé de silex que met en saillie la dénudation de la craie. En approchant de la basse mer, le lit du ruisseau s'élargit et devient sablonneux. Le courant est bien moins considérable que celui de la source $\mathrm{n}^{\circ} 2$; cependant le volume d'eau, au point de sortie, ne suffit pas pour en justifier l'importance et, sans nul doute, d'autres orifices existent dans son lit.

A l'crigine même de la source se développe une abondante végétation de Ceramium rubrum Cladophora rupestris, Ulva Lactuca $f$ contorta et Porphyra laciniata. Les deux premières de ces plantes acquièrent ici de très grandes dimensions. Plus bas se rencontrent en grosses touffes les Gigartina mamillosa, Chondrus crispus, Cladophora rupestris, Phyllophora membranifolia (2). Cette dernière espèce atteint dans l'eau douce des proportions que je n'ai rencontrées nulle part ailleurs.

Le Gracilaria confervoides n'est pas rare dans la région que j'ai explorée, mais il croìt exclusivement sur les fond sablonneux, principalement dans les petits courants qui servent d'écoulement aux flaques laissées par la mer. Il est à remarquer que je ne l'ai rencontré ni dans la source $n^{\circ} 2$, ni dans la source $n^{\circ} 4$, bien que les ruisseaux de décharge de l'une et de l'autre, coulant sur un lit de sable, rentrent absolument dans la catégorie des localités

(1) Sussette suivant les cartes marines.

(2) Suivant Debray, les cystocarpes de ce Phyllophora ne se développeraient abondamment que dans les endroits où coule l'eau douce. Je n'ai pas constaté l'exaclitude de cette assertion qui ne semble pas d'accord avec ce qu'on observe chez la plupart des plantes. On sait en effet que le développement des organes végétatifs est, en général, l'inverse de celui des organes reproducteurs.

Conf. Debray, loco cit., p. 108. 
GOMONT. - VÉGÉTATION DES SOURCES D'EAU DOUCE SOUS-MARINES. 45 oủ se plaìt la plante. Toutefois la présence de cette espèce sur les fonds de pareille nature n'est pas assez constante pour que son absence puisse être attribuée d'une manière certaine à l'influence de l'eau douce.

Les plantes trouvées dans la source $\mathbf{n}^{\circ}$ / sont les suivantes:

Ceramium rubrum.

Chondrus crispus.

Claduphora rupestris.

Entoromorpha intestinalis. Ulva Lactuca forma contorta.

Porphyra laciniata.

Phyllophora membranifolia. Cladophora utriculosa.
Gigartina mamiljosa.

Gelidium crinale.

Ceramium Deslongchampsii.

Nitophyllum laceratum.

Laurencia pinnatifida.

Furcellaria fastigiata.

Corallina officinalis.

Les cinq dernières espèces n’étaient représentées que par un très petit nombre d'échantillons et, mème, le Corallina officinalis par un seul très peu développé. Je n’en ai d'ailleurs rencontré aucun autre dans les mêmes circonstances.

\section{Source $\mathbf{n}^{\circ} \mathbf{5}$.}

Elle sort, à 500 mètres environ du vallon de Sunsette, entre ce dernier et la grande vallée de la Durdent. Son orifice se trouve d peu près à la même distance du niveau supérieur des marées que celui de la source précédente, entre de gres blocs de grès provenant de la couche superficielle de la falaise; il n'existe en ce point ni dépôt calcaire meuble, ni sable. Bien que le débit de cette source soit assez faible, elle ne laisse pas que de former un ruisseau sinueux dont on peut suivre le cours jusqu'à la basse mer. Il coule tantôt sur des calcaires polis, tantôt sur un lit de sable qui devient fortement vaseux dans les petits bassins oủ le courant se ralentit. L'Enteromorpha intestinalis et le Cladophora rupestris forment à eux seuls la végétation de ces bassins. $\mathrm{Au}$ milieu mème $\mathrm{du}$. courant se rencontre le Gallithamnion Hookeri, comme dans la source $\mathrm{n}^{\circ} 2$, seulement il est ici parasite sur le Cladophora rupestris au lieu d'être fixé sur des Floridées.

Les plantes observées dans la source $n^{\circ} 5$ sont les suivantes:

Enteromorpha intestinalis.

Ulva Lactuca.

Porphyra laciniata.

Cladophora rupẹstris.

Ceramium rubrum.
Chondrus crispus.

Callithamnion Hookeri.

Cladophora flexuosa.

Dumontia filiformis. 
Cette dernière espèce n'a été rencontrée qu'une seule fois. L'échantillon était peu développé.

Source $\mathrm{n}^{\circ} 6$.

Si, poursuivant sa route dans la direction que nous avons adoptée, on traverse la vallée de la Durdent, puis qu'on gravisse la falaise pour en suivre le sommet, on rencontre, à la hauteur du village de Saint-Martin-aux-Buneaux, un pli de terrain perpendiculaire à la côte et aboutissant à une profonde fissure de la muraille calcaire. L'étroit sentier qui s'y engage débouche à une certaine hauteur au-dessus de la mer, à laquelle on accède par des échelles fixées au rochęr. Au bas de celles-ci, c'est-à-dire tout à fait à la limite supérieure des marées, une source assez abondante alimente un petit bassin creusé dans les galets du cordon littoral, et utilisé par les femmes du pays pour laver leur linge pendant le temps du reflux. Au-dessous, l'eau douce forme un ruisseau qui coule sur un lit de sable et se divise en plusieurs bras, couvrant un espace étendu. Aucune végétation dans le bassin même, ce qu'explique facilement l'usage auquel il est employé; mais, un peu au-dessous, le lit de sable est couvert d'une abondante prairie d'Enteromorpha intestinalis. Au milieu se trouvent des blocs émergés portant des touffes nombreuses de Fucus vesiculosus et de Cladophora rupestris; mais, là comme ailleurs, Fucacées et Corallines manquent sur les points soumis, durant le reflux, à l'influence permanente de l'eau douce. On les retrouve en revanche un peu plus bas, là où le courant se mêle aux nombreuses flaques salées laissées par la mer. En résumé, la végétation est ici ee que nous la trouvons sur tous les points où elle est soumise pendant longtemps à l'action de l'eau douce, en d'autres termes, là oủ les sources se montrent au voisinage de la limite des hautes mers.

Il en est ainsi par exemple d'une fontaine qui se rencontre à peu de distance en aval (1) de Fécamp et à laquelle je ne consacrerai pas un chapitre spécial, pour éviter d'inutiles rediles. Elle

(1) Les habitants du littoral de la Seine-Inférieure, appellent amont la direction de la mer du Nord et aval la direction de l'Atlantique. Cette expression est parfaitement justifiée, il existe en effet un courant qui se dirige du Pas-de-Calais vers l'Océan. 
ne rentre pas d'ailleurs dans le cadre de cette étude, mais plutòt dans la catégorie des ruisseaux còliers, car elle sort de la falaise à quelques mètres au-dessus du niveau des plus hautes marées. Après avoir glissé sur un plan très incliné, bordé d'un verdoyant tapis de mousses, elle atteint le galet, où elle est reçue dans un bassin et employée aux usages domestiques. Comme la source précédente, elle provoque dans les environs une abondante végétation d'Enteromorpha.

\section{Sources $\mathrm{n}^{\circ \mathrm{s}} 7$ et 8 .}

De toutes celles que nous avons explorées, ce sont de beaucoup les plus connues, leur situation sur les deux plages très fréquentées d'Yport et d'Étretat attirant sur elles l'attention de ceux mêmes que l'étude des Algues n'intéresse à aucun degré. A mon grand regret, je n'ai visité Yport qu'à une epoque où je ne m'occupais pas spécialement de la végétation des sources. Celle d'Yport a été captée pour les besoins de la petite ville depuis mon voyage, de sorte que je n'aurais rien à en dire si je n'y avais récolté à cette époque le Callithamnion Hookeri, déjả mentionné dans les listes précédentes, et le Fucus ceranoides qui, au contraire, n'y figure pas et que je n'ai rencontré sur aucun autre point de la côte. Je l'ai notamment cherché avec attention, mais sans succès, à l'embouchure de la Durdent, dans le but d'essayer l'acclimatation de cette Fucacée aux abords des sources d'eau douce où on est étonné de ne pas la trouver. D'ailleurs Debray ne la cile qu'à Fécamp, à Yport et à Port-en-Bessin. Celte dernière localité, située dans le Calvados, n'appartient pas à notre région.

On a parlé plus souvent encore de la source d'Étretat ( $\left.\boldsymbol{n}^{\circ} 8\right)$, qui serait, dit-on, le dernier vestige d'une rivière disparue dans le sol (1). Que le fait soit exact ou non, une nappe d'eau douce coule aetuellement sous les galets, en un point de la zone littorale, comme le prouve l'usage qu'ont les lavandières du pays d's creuser en guise de baquets, pendant les basses mers, des excavations qui se remplissent instantanément. Au bas des galets, l'eau se montre à l'air libre, s'étendant sur un large espace couvert de sable vaseux. Dans cette sorte de petit estuaire, nous

(1) Conf. Passy, Description géologique du département de la Seine-Inférieure, p. 30, 1832. 
trouvons, ainsi que partout ailleurs dans les mèmes conditions, l'Enteromorpha intestinalis comme espèce prépondérante. Avec lui, mais en quantité moindre, se trouvent les Cladophora rupestris, Chondrus crispus, Gigartina mamillosa, ainsi que quelques touffes de Fucus serratus assez maigres. Je n'y ai pas vu de Corallines. La nappe d'eau douce est limitée d'un côté par un banc de roches plates, situées hors de son atteinte et recouvertes par le tapis habituel de Fucus. L'examen de cette source ne fait donc que confirmer ce que nous savons déjà et, comme chez toutes celles de même niveau, le nombre des espèces y est peu considérable.

Mes investigations n'ont pas dépassé Étretat, mais l'uniformité des résultats obtenus, celle de la Flore générale de la région, la similitude des localités où se montre l'eau douce, me donnent à penser que des recherches poussées plus loin, du moins sur la côte de la Seine-Inférieure, n'ajouteraient rien à ce que nous avons appris.

En revanche, il serait intéressant de comparer ces données avec celles que fournirait l'exploration de la còte opposée de la baie de la Seine, dont la végétation marine est différente, et qui appartient à une autre formation géologique.

Avant d'exposer en le résumant ce qui ressort de l'examen détaillé que nous venons de faire de chacune des sources explorées, il importe de rechercher à quel régime se trouvent soumises au point de vue de la salure des eaux, les plantes que nous y avons rencontrées.

Ces sources, comme on a pu le remarquer, appartiennent à deux catégories différentes. Les unes $\left(n^{\circ 8} 3,6,8\right)$, sortent du sol tout près de la falaise, soit dans le cordon littoral de galets, soit à son bord inférieur, par conséquent au niveau supérieur des marées, ou peu s'en faut; les autres $\left(n^{o s} 1,2,4,5\right)(1)$ à une distance qui varie de 100 à 200 mètres du pied de la falaise, c'està-dire à peu près à mi-marée. Elles ne se montrent donc pas à tous les niveaux, et il est à remarquer notamment que je n'en ai point rencontré à celui des basses mers. Les gens du pays qui, par profession, fréquentent continuellement la plage, n'ont pu

(1) La source $n^{\circ} 7$ (Yport) appartient à celte catégerie. Je ne la mentionne pas, ne l'ayant pas explorée suffisamment, comme on l'a vu plus haut. 
d'ailleurs m'en indiquer aucune en cet endroit. Le fait n'a rien de surprenant puisque la formation des nappes d'eau douce dépend de la situation des couches de terrain imperméables, laquelle varie peu sur une étendue restreinte comme celle que nous avons parcourue. La règle que nous indiquons ici est toute locale, cela va sans dire; les niveaux d'eau douce et, par conséquent, les points de sortie des sources sous-marines, diffèrent évidemment suivant les régions considérées.

La végélation des deux catégories de sources que nous venons d'indiquer ne saurait ètre identique. D'une part, en effet, elle est soumise aux lois qui règlent sur un même point la répartition des espèces, car on sait, que celles-ci varient avec la profondeur; de l'autre, le temps pendant lequel s'exerce l'influence de l'eau douce diffère considérablement suivant le niveau où elle sort. Il n'est pas d'ailleurs proportionnel à la distance qui sépare ce niveau de la limite des basses mers. Pour qu'il en fût ainsi, il faudrait que le rivage formât une pente uniforme, tandis qu'il se divise, comme nous l'avons vu, en deux zones, l'une étroite et fortement inclinée, l'autre beaucoup plus large et sensiblement horizontale. La marée couvre celle-ci en deux heures environ, tandis que sa marche ascendante devient très lente dès qu'elle atteint le cordon littoral de galets. Aussi ne peut-on guère évaluer à plus de quatre heures par jour le temps pendant lequel les plantes des sources à mimarée seront soumises au régime de l'eau douce, alors qu'il atteindra huit heures pour celles qui se trouvent à la base du talus de galets. Quant aux sources situées au pied mème des falaises, comme celle de Saint-Martin-aux-Buneaux ( $\left.n^{\circ} 6\right)$, elles ne seront couvertes par l'eau de mer que pendant quelques instants:

Remarquons que, pour toutes ces sources, quel que soit leur niveau, il existe un facteur dont il importe de tenir compte; je veux parler de leur température. Sauf peut-être pendant l'hiver, elle reste plus basse que celle de la mer ou des flaques environnanteș. Je dirai même que c'est à cette différence, facilement appréciable au toucher, que je dois d'avoir remarqué pour la première fois les courants d'eau douce qui font l'objet de ce travail. Avec le thermomètre j'ai trouvé les résultats suivants : le 9 septembre dernier, à la fin d'une journée où le ciel avait été presque constamment couvert et par un vent violent du sud-ouest, 
la température de l'air étant de 16 degrés, j'ai trouvé pour celle des sources $\mathrm{n}^{\circ \mathrm{s}} 2$ et 4,12 degrés et $12^{\circ}, 2$. Au même moment, celle de la mer était de $14^{\circ}, 6$ et celle des flaques de $14^{\circ}, 5$. Cette différence de $2^{\circ}, 5$ environ, qui est déjà notable, ne doit d'ailleurs être regardée que comme un minimum. Si, au lieu d'être faite par un temps froid et couvert, l'observation l'eût été à la fin d'une journée chaude et ensoleillée, la température de la mer et surtout celle des flaques stagnantes eût été beaucoup plus élevée, tandis que celle des sources serait restée, sans nul doute, sensiblement la même. On sait en effet que la température de l'air influe peu, en général, sur celle de ces dernières, surtout si elles sont issues de nappes profondes.

Il est vrai qu'étant donné le volume très faible de l'eau douce relativement à celui de la mer, cette différence doit être annihilée pendant le temps du flux. Néanmoins les Algues soumises à l'influence des sources éprouvent constamment l'effet d'une température sensiblement uniforme, mais relativement basse, tandis que celles qui vivent dans les mares salées jouissent d'une température qui, en certaines saisons et pendant quelques heures de la journée, peut s'élever beaucoup plus haut. Le régime est donc très différent dans les deux cas et moins favorable à la multiplicité des espèces dans celui que nous étudions en particulier, puisque nous voyons la richesse de la Flore marine, comme celle de la Flore terrestre, décroître avec la chaleur du climat.

Nous donnerons maintenant la liste des plantes dont nous avons noté la présence, avec l'indication du nombre de sources où chacune a été rencontrée. Elle montrera comment agissent sur les différentes espèces les conditions biologiques spéciales que nous venons d'exposer.

Enteromorpha intestinalis, rencontré dans 6 sources.

Cladophora rupestris,

Ulva Lactuca,

Chondrus crispus,

Ceramium rubrum,

Porphyra laciniata,

Gigartina mamillosa,

Callithamnion Hookeri,

Ceramium Deslongchampsii,

Pbyllophora membranifolia,

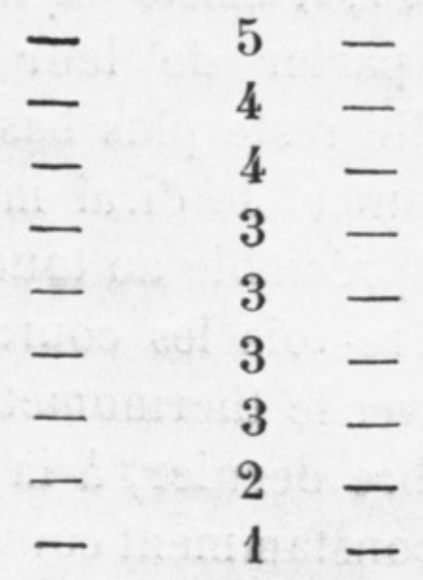




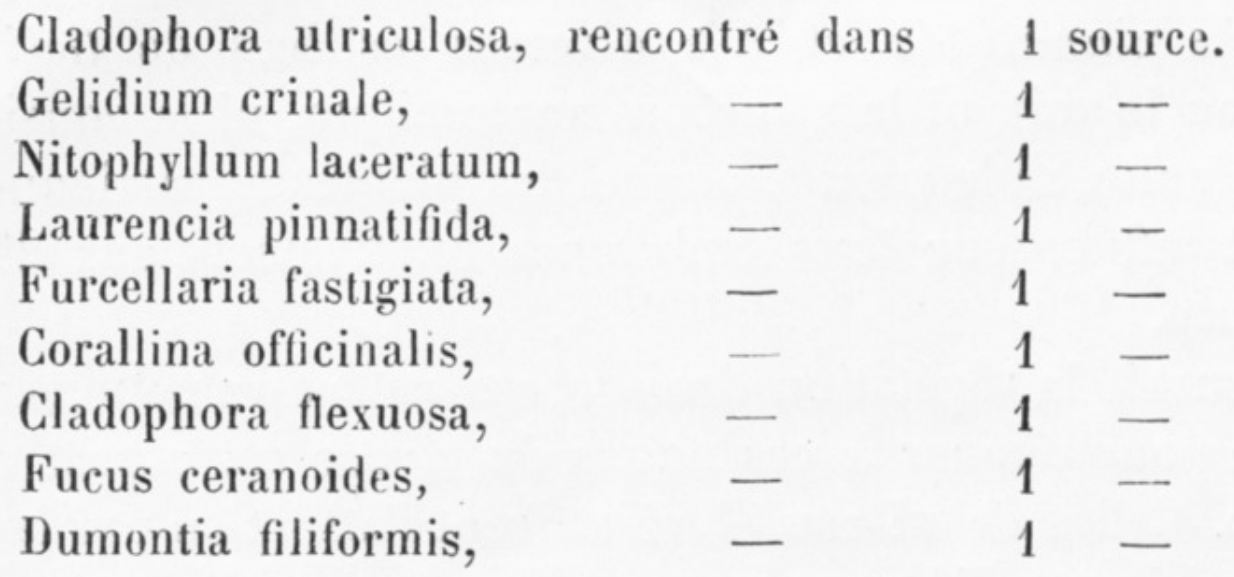

Quelques observations seront à leur place à la suite de cette liste.

Je dirai d'abord que les huit premières espèces doivent seules, à mon avis, être regardées comme caractéristiques. Elles ont été trouvées, en effet, dans toutes les sources dont le niveau répondait à leurs exigences biologiques et elles y figuraient en échantillons nombreux et vigoureusement développés. Au contraire les autres n'ont été rencontrées chacune que dans une seule localité et elles n'étaient représentées que par un petit nombre d'individus, souvent même par un seul, de chétive apparence. On doit, je pense, regarder la présence de celles-ci comme accidentelle et leur nombre pourra être augmenté par de nouvelles recherches. Je crois cependant devoir distraire de ces espèces négligeables le Phyllophora membranifolia et le Fucus ceranoides. Le premier n'a été trouvé, il est vrai, que dans la source de la Pêcherie $\left(n^{\circ} 4\right)$, mais en échantillons nombreux et remarquablement développés. Quant au Fucus ceranoides, sa rareté dans les localités que nous envisageons particulièrement ici s'explique par son peu de fréquence dans la région d'une manière générale, mais il est l'hòte si habituel des eaux saumâtres qu'on ne saurait l'exclure sans invraisemblance de. la liste des espèces caractéristiques.

En second lieu, on remarquera que, parmi ces dernières, manquent un certain nombre d'espèces des plus répandues sur tout ce littoral et d'ordinaire extrêmement abondantes dans les localités où s'épanchent les sources d'eau douce de la deuxième catégorie. Ainsi, outre l'absence si frappante des Fucus et des Corallines, sur laquelle nous avons déjà attiré l'attention, nous signalerons celle des Rhodochorton floridulum, Plocamium coccineum, Cystoclonium purpurascens, Nitophyllum laceratum, 
Laurencia pinnatifida et des diverses espèces de Gelidium. Comme on le voit, ni la structure anatomique, ni la consistance qui en résulte, ni le groupe auquel les plantes appartiennent ne semblent influer sur leur endurance relativement aux effets de l'eau douce.

En résumé, la végétation des sources est assez nettement différenciée pour donner à ces points du rivage un aspect reconnaissable. Celles de niveau élevé attirent l'attention par la végétation exubérante d'Algues vertes, Ulvacées et Cladophoracées, qu'elles provoquent. Quant aux sources de mi-marée, leur point de sortie nous montre aussi des Ulvacées, mais moins abondantes (1). En revanche, il est le plus souvent obstrué par des touffes volumineuses de Ceramium rubrum et de Cladophora rupestris auxquelles viennent se joindre les membranes délicates du Porphyra laciniata. En descendant vers la mer, on voit le courant d'eau douce se garnir de Gigartinées, Chondrus crispus, Phyllophora membranifolia, Gigartina mamillosa, dont la couleur, d'un noir rougeàtre, tranche sur celle de la végétation environnante. Aussi l'œil s'habitue-t-il aisément, avec quelque exercice, à reconnaître presque à coup sûr la présence d'un courant d'eau douce. Je ne suis pas d'ailleurs le seul qui les ait remarqués et, comme j'ai pu m'en convaincre, les pêcheurs qui fréquentent le rivage pendant la basse mer, n'en ignorent, pour la plupart, ni l'existence, ni la situation. J'ajouterai même que les plus observateurs d'entre eux ont été frappés de l'aspect insolite de la végétation qu'ils engendrent.

On aura peut-être quelque peine à croire que des quantités d'eau douce si insignifiantes relativement à la masse de l'eau salée, puissent amener une modification appréciable dans la végétation. Ce phénomène surprendra surtout chez les sources situées à mi-marée, où la durée d'action s'exerce pendant un temps très court que nous avons évalué à quatre heures par jour seulement.

(1) Bien que la source du Trou au vin ( $\left.\mathrm{n}^{\circ} 2\right)$, soit placée fort au-dessous du niveau supérieur des marées et appartienne par conséquent à la deuxième catégorie, il est à remarquer que son orifice et la région environnante sont tapissés d'Enteromorpha, comme chez les sources du cordon littoral. Je ne puis attribuer cette anomalie qu'à son débit beaucoup plus considérable que celui de toutes les autres de même niveau. On peut admettre que le volume de l'eau douce et la durée de son action produisent, en cette circonstance, des effets identiques. 
GOMONT. - VÉGÉTATION DES SOURCES D'EAU DOUCE SOUS-MARINES. 53

Nous pensons cependant que le travail de M. Oltmanns, cité au début de cette Note, peut donner du fait une explication plausible.

L'auteur, prenant pour matériaux d'étude le Fucus vesiculosus el le Polysiphonia nigrescens, a institué deux séries de cultures dont les unes conservaient la même eau pendant toute la durée de l'expérience, tandis que les autres recevaient de l'eau nouvelle dont la salure était différente. Dans le premier cas, les plantes ont gardé leur aspect et leur rapidité de développement normaux; dans le second, au contraire, elles ont présenté des altérations dans leur couleur et dans leur reproduction, ainsi qu'un ralentissement notable de leur allongement quotidien (1). Toutefois l'action nuisible ne s'est pas manifestée lorsque l'eau était ajoutée graduellement, au lieu d'être versée d'un seul coup.

Des observations comparatives faites sur des points de la Baltique oú la salure ne varie pas sensiblement et sur d'autres où elle change à chaque marée, ont confirmé les expériences de laboratoire. L'auteur a donc pu dire avec raison que les modifications qui se produisent dans la teneur en sel de l'eau de mer exercent sur les plantes une influence nuisible et d'autant plus marquée qu'elles ont lieu plus brusquement, de sorte que, dans les localités oủ elles sont la règle, la Flore sera pauvre comparativement à celles où la salure des eaux demeure constante. Enfin, chose importante à noter, l'action nocive de ces changements n'a, suivant M. Oltmanns, aucune relation avec les fonctions de nutrition, les proportions des matières dissoutes étant toujours supérieures aux besoins de la plante; cette action dépend uniquement des changements produits dans la turgescence de la cellule (2).

Les faits relatés ci-dessus et ceux que nous avons observés nous-même présentent, comme on le voit, de grands rapports dans leur ensemble, et la même explication doit convenir aux uns et aux autres. Il est' difficile, en effet, d'imaginer un changement de milieu plus brusque et plus absolu que celui qu'éprouvent les plantes des sources au moment où la marée les courre ou les décoúvre. Dans un intervalle de quelques minutes elles passent, en effet, de l'eau douce à celle de la Manche ou inversement, et

(1) V. Oltmanns, loco cit., p. 195.

(2) V. Oltmanns, loco cit., p. 203. 
l'on sait que la salure de cette mer est beaucoup plus forte que celle de la Baltique où $\mathrm{M}$. Oltmanns a recueilli ses observations. $\mathrm{Si}$ ces alternatives n'influaient que sur la nutrition, il serait difficile de comprendre, du moins pour les sources de mi-marée, que la privation de certains éléments, pendant un nombre d'heures aussi restreint, puisse amener la disparition ou l'étiolement d'une proportion notable d'espèces. On peut admettre, au contraire, sans difficulté, qu'une modification brusque dans la turgescence, répétée quatre fois en vingt-quatre heures, produise, à la longue, une altération de la cellule, capable de conduire aux résultats que nous avons constatés. En effet, l'immersion dans l'eau douce amène la destruction immédiate de beaucoup d'Algues marines; le contenu cellulaire se décompose et souvent la membrane se rompt. Il suffit même d'une pluie abondante pendant que la mer est basse pour en faire périr un grand nombre. D'autres, moins altérables, subissent des modifications plus ou moins profondes qui en diminuent la vitalité.

Toutefois cette hypothèse, il faut le reconnaître, n'explique aucunement le développement insolite observé chez quelques espèces: Ceramium rubrum, Cladophora rupestris, diverses Gigartinées, et ce fait semble même contredire la théorie de M. Oltmanns. Comme plusieurs de ces plantes, telles que les Phyllophora membranifolia, Gigartina mamillosa, Chondrus crispus, abondent surtout dans l'Atlantique septentrional, je serais disposé à chercher la cause de cette végétation luxuriante dans l'action continue d'une basse température qui compenserait, et au delà, l'influence fâcheuse des fréquents changements de concentration du milieu. Il est en effet inadmissible que des espèces essentiellement marines, comme celles que nous venons de citer, rencontrent dans les sources des èléments nutritifs utiles qui leur manquent dans leur milieu naturel. Nous trouvons d'ailleurs dans les travaux de M. Darbishire la preuve manifeste des préférences nettement halophiles de certaines Gigartinées (1), car il a décrit et figuré des formes appauvries de plusieurs Phyllophora, dont la dégénérescence ne peut être attribuée qu'à la faible salure des eaux de la Baltique où elles ont été observées.

(1) 0. V. Darbishire, Die Phyllophora-Arten der Westlichen Ostsee deutschen Antheils, pp. 5-10. 
Du reste le Mémoire de cet auteur et ceux de M. Reinke sur la Flore marine des environs de Kiel (1), semblent montrer que l'effet physiologique produit sur les Algues par un milieu dont la teneur en sel est faible, mais constante, n'est pas comparable à celui qui résulte de variations de salure brusques et fréquentes comme celles que nous avons décrites.

MM. Molliard pense que les effets de la plasmolyse pourraient expliquer la présence ou l'absence de certaines Algues dans des eaux alternativement douces et salées.

M. de Boissieu fait la communication suivante :

\section{SUR QUELQUES PLANTES ADVENTICES DE L'AIN ;}

\section{par M. H. de Boissieu.}

Nous avons poursuivi en 1903, nos recherches dans la localité voisine de Pont-d'Ain, dont nous avions l'an dernier l'honneur d'entretenir la Société (séance du 27 février 1903), où, grâce aux déchets, aux graines de rebut d'une grande minoterie, se crée peu à peu une véritable florule spéciale. A la liste des espèces adventices de Pont-d'Ain donnée précédemment par nous il convient d'ores et déjà d'ajouter les trois plantes suivantes :

Gypsophila paniculata L.

Centaurea solstitialis L. et le curieux hybride Centaurea diffusa $\times$ Jacea ou C. juvenalis Del.

Ce produit, dont nous connaissons pour le moment deux pieds, se présente, à Pont-d'Ain, sous une forme peu différente de celle de Port-Juvénal, et nos échantillons rappellent tout à fait ceux, par exemple, provenant des doubles de l' «Herbier méditerranéen 》. Les organes de végétation sont à peu près ceux d'un $C$. Jacea, sauf pour les feuilles inférieures, qui sont pennati-ou bipennatiséquées. Les capitules ont la grosseur de ceux du C. Jacea; .es fleurons sont tantôt roses, tantòt blancs, tantòt mélangés, les extérieurs roses, les intérieurs blancs. Les écailles involucrales sont presque celles d'une forme pâle de C. Jacea; puis, sans tran sition, les écailles extérieures deviennent à peu près celles d'un C. diffusa var. brevispina.

(1) J. Reinke, Algenflora der Westlichen Ostsee deutschen Antheils, 1889 ; Atlas deutschen Meeresalgen, Erstes Heft, 1889. 


\section{$2 \mathrm{BHL}$ Biodiversity Heritage Library}

Gomont, Maurice. 1904. "Sur La Végétation De Quelques Sources D'Eau Douce Sous-Marines De La Seine-Inférieure." Bulletin de la Société botanique de France 51, 36-55. https://doi.org/10.1080/00378941.1904.10829084.

View This Item Online: https://www.biodiversitylibrary.org/item/8673

DOI: https://doi.org/10.1080/00378941.1904.10829084

Permalink: https://www.biodiversitylibrary.org/partpdf/160315

\section{Holding Institution}

Missouri Botanical Garden, Peter H. Raven Library

\section{Sponsored by}

Missouri Botanical Garden

\section{Copyright \& Reuse}

Copyright Status: Public domain. The BHL considers that this work is no longer under copyright protection.

This document was created from content at the Biodiversity Heritage Library, the world's largest open access digital library for biodiversity literature and archives. Visit BHL at https://www.biodiversitylibrary.org. 